\title{
An Experimental Investigation to Compare Heat Augmentation From Plane And Protruded Rectangular Fins
}

\author{
Associate Proff. Anirudh Gupta ${ }^{1,}$ Pramod Bhatt ${ }^{2}$ \\ ${ }^{I}$ (BTKIT, Dwarahat $)^{2}($ M.Tech Scholar $)$
}

\begin{abstract}
An investigation is to be conducted to determine whether protrusion on a fin can increase heat transfer under forced convections or not. This would be accomplished by performing an experimental investigation. This research investigates new fin topologies with heat transfer characteristics that will surpass conventional fin, where the main goals are to increase the heat transfer rate of the fin surface while keeping an acceptable pressure drop penalty. Boundary layer regeneration and enhanced flow mixing are the main techniques through which fins increase the overall Nusselt number of the surface. Surface roughness is usually applied to smooth surfaces to promote flow mixing and initiate turbulence in the flow. In this experiment the radiation factor is neglected. Increasing the temperature difference between the object and the environment, increasing the convection heat transfer coefficient, or increasing the surface area of the object increases the heat transfer. Sometimes it is not economical or it is not feasible to change the first two options. Adding a fin to an object, however, increases the surface area and can sometimes be an economical solution to heat transfer problems.
\end{abstract}

Keywords - Protrusion, Sand Grain Size, Nusselt Number, Augmentation, Turbulence

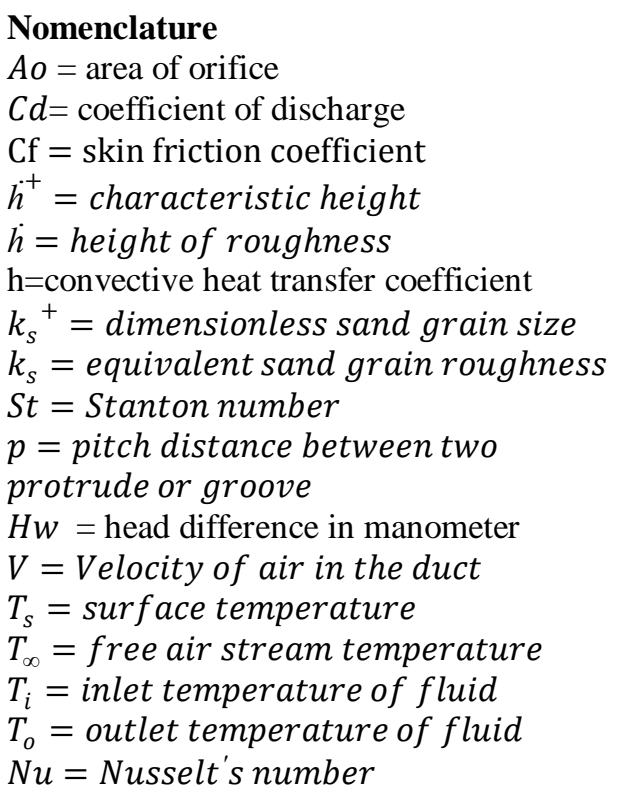

\section{INTRODUCTION}

For well over a century, efforts have been made to produce more efficient heat exchangers by employing various methods of heat transfer enhancement. The study of enhanced heat transfer has gained serious momentum during recent years, however, due to increased demands by industry for heat exchange equipment that is less expensive to build and operate than standard heat exchange devices. Savings in materials and energy use also provide strong motivation for the development of improved methods of enhancement. When designing cooling systems for automobiles and spacecraft, it is imperative that the heat exchangers are especially compact and lightweight. Also, enhancement devices are necessary for the high heat duty exchangers found in power plants (i.e. air-cooled condensers, nuclear fuel rods).These applications, as well as numerous others, have led to the development of various enhanced heat transfer surfaces. The latter is particularly useful in thermal processing of biochemical, food, plastic, and pharmaceutical media, to avoid thermal degradation of the 
end product. On the other hand, heat exchange systems in spacecraft, electronic devices, and medical applications, for example, may rely primarily on enhanced thermal performance for their successful operation. The commercialization of enhancement techniques, where the technology has been transferred from the research laboratory to full-scale industrial use of those that are more effective and, has also led to a larger number of patents.

The conversion, utilization, and recovery of energy in every industrial, commercial, and domestic application involve a heat transfer process. Some common examples are coming from domestic application to industrial ones. Improved heat exchange, over and above that in the usual or standard practice, can significantly improve the thermal efficiency in such applications as well as the economics of their design and operation. The engineering cognizance of the need to increase the thermal performance of heat based equipments, thereby effecting energy, material, and cost savings as well as a consequential mitigation of environmental degradation had led to the development and use of many heat transfer enhancement techniques. These methods have in the past been referred to variously as augmentation and intensification, among other terms.

In general, enhanced heat transfer surfaces can be used for three purposes: to make heat exchangers more compact in order to reduce their overall volume, and possibly their cost, to reduce the pumping power required for a given heat transfer process, or to increase the overall UA value of the heat exchanger.

A higher UA value can be exploited in either of two ways:

(1) To obtain an increased heat exchange rate for fixed fluid inlet temperatures, or

(2) To reduce the mean temperature difference for the heat exchange; this increases the thermodynamic process efficiency, which can result in a saving of operating costs. Enhancement techniques can be separated into two categories: passive and active. Passive

Methods require no direct application of external power. Instead, passive techniques employ

Special surface geometries or fluid additives which cause heat transfer enhancement. On the other hand, active schemes such as electromagnetic fields and surface vibration do require external power for operation. The majority of commercially interesting enhancement techniques are passive ones. Active techniques have attracted little commercial interest because of the costs involved, and the problems that are associated with vibration or acoustic noise. This paper deals only with gas-side heat transfer enhancement using special surface geometries. Special surface geometries provide enhancement by establishing a higher hA per unit base surface area.

\section{CLASIFICATION OF ENHANCEMENT TECHNIQUES}

Heat transfer enhancement or augmentation techniques refer to the improvement of thermo hydraulic performance of heat exchangers. Existing enhancement techniques can be broadly classified into three different categories:

1 Passive Techniques

2 Active Techniques

3 Compound Techniques.

The effectiveness of any of these methods is strongly dependent on the mode of heat transfer (singlephase free or forced convection, pool boiling, forced convection boiling or condensation, and convective mass transfer), and type and process application of the heat exchanger.

PASSIVE TECHNIQUES: These techniques generally use surface or geometrical modifications to the flow channel by incorporating inserts or additional devices. They promote higher heat transfer coefficients by disturbing or altering the existing flow behaviour (except for extended surfaces) which also leads to increase in the pressure drop. In case of extended surfaces, effective heat transfer area on the side of the extended surface is increased.

Passive techniques hold the advantage over the active techniques as they do not require any direct input of external power. Heat transfer augmentation by these techniques can be achieved by using:

Treated Surfaces: This technique involves using pits, cavities or scratches like alteration in the surface of the heat transfer area which may be continuous or discontinuous. They are primarily used for boiling and condensing duties.

Rough surfaces: These surface modifications particularly create the disturbance in the viscous sub-layer region. These techniques are applicable primarily in single phase turbulent flows.

Extended surfaces: Plain fins are one of the earliest types of extended surfaces used extensively in many heat exchangers. Finned surfaces have become very popular now days owing to their ability to disturb the flow field apart from increasing heat transfer area.

Displaced enhancement devices: These inserts are used primarily in confined forced convection. They improve heat transfer indirectly at the heat exchange surface by displacing the fluid from the heated or cooled surface of the duct with bulk fluid from the core flow. 
Swirl flow devices: They produce swirl flow or secondary circulation on the axial flow in a channel. Helical twisted tape, twisted ducts \& various forms of altered (tangential to axial direction) are common examples of swirl flow devices. They can be used for both single phase and two-phase flows.

Coiled tubes: In these devices secondary flows or vortices are generated due to curvature of the coils which promotes higher heat transfer coefficient in single phase flows and in most regions of boiling. This leads to relatively more compact heat exchangers.

Surface tension devices: These devices direct and improve the flow of liquid to boiling surfaces and from condensing surfaces. Examples include wicking or grooved surfaces.

Additives for liquids: This technique involves addition of solid particles, soluble trace additives and gas bubbles added to the liquids to reduce the drag resistance in case of single phase flows. In case of boiling systems, trace additives are added to reduce the surface tension of the liquids.

ACTIVE TECHNIQUES: These techniques are more complex from the use and design point of view as the method requires some external power input to cause the desired flow modification and improvement in the rate of heat transfer. It finds limited application because of the need of external power in many practical applications. In comparison to the passive techniques, these techniques have not shown much potential as it is difficult to provide external power input in many cases. Various active techniques are as follows:

Mechanical Aids: Examples of the mechanical aids include rotating tube exchangers and scrapped surface heat and mass exchangers. These devices stir the fluid by mechanical means or by rotating the surface.

Surface vibration: They have been used primarily in single phase flows. A low or high frequency is applied to facilitate the surface vibrations which results in higher convective heat transfer coefficients.

Fluid vibration: Instead of applying vibrations to the surface, pulsations are created in the fluid itself. This kind of vibration enhancement technique is employed for single phase flows.

Electrostatic fields: Electrostatic field like electric or magnetic fields or a combination of the two from DC or $\mathrm{AC}$ sources is applied in heat exchanger systems which induces greater bulk mixing, force convection or electromagnetic pumping to enhance heat transfer. This technique is applicable in heat transfer process involving dielectric fluids.

Injection: In this technique, same or other fluid is injected into the main bulk fluid through a porous heat transfer interface or upstream of the heat transfer section. This technique is used for single phase heat transfer process.

Suction: This technique is used for both two phase heat transfer and single phase heat transfer process. Two phase nucleate boiling involves the vapour removal through a porous heated surface whereas in single phase flows fluid is withdrawn through the porous heated surface.

Jet impingement: This technique is applicable for both two phase and single phase heat transfer processes. In this method, fluid is heated or cooled perpendicularly or obliquely to the heat transfer surface.

COMPOUND TECHNIQUES: A compound augmentation technique is the one where more than one of the above mentioned techniques is used in combination with the purpose of further improving the thermo-hydraulic performance of a heat exchanger

\section{LITERATURE REVIEW}

In a study for a given base temperature, the effect of vacuum, angle of groove and number of thread per inch shows that there exist optimum angle of groove and number of thread per inch for which the heat loss per unit mass is maximum.i.e; heat loss is 1.2 to 1.4 times greater than radiating pin fin [1]. The performance of heat exchanger can be improved by mounting protrusion on the surface. The rectangular winglet mounted on the triangular fins of the plate fin heat exchanger disturbs the flow structure and creates longitudinal vortices. Due to the existence of complex stream wise vortices system in the flow passage, the heat transfer between the fluid and its neighboring surface is significantly enhanced with a moderate pressure drop [2]. It has been found that the grooved radiating fin loses approximately 1.23 times greater heat per unit area compared to the threaded fin. As pressure decreases heat loss reduces and contribution of radiation heat transfer on total heat loss increases [3]. Study in different vortex generator shows that more complicated fin structure will lead to higher pressure drop and the pressure drop for dimple fin geometry is significantly higher than other fin types. The test result of heat transfer coefficient suggested that fin with dimple vortex generator is more beneficial than that of plain fin [5]. Study of dimpled surface with uniform heat flux shows that heat transfer coefficient are relatively low on the leading edge of the dimple and high on the trailing edge and the flat area immediately downstream of the dimple [6]. In numerical optimization of heat exchanger with brazed dimpled surface investigate friction factor and colburn factor according to parameter. The heat transfer and pressure drop increases as the dimpled diameter and channel height increases while they decreases as the dimple spacing and dimple pitch increased [7]. The transverse distance had a greater effect on the enhanced heat transfer than the stream wise distance is 
shown by study on pin fin array with turbulent air flow [8]. Natural convection heat transfer enhancement from a horizontal rectangular fin embedded with equilateral triangular perforation is investigated and compared with equivalent solid one. The gain in heat dissipation rate for perforated fin has strong relation with perforation dimension and lateral spacing [9]. Mills and Ganesan also gave relation for heat transfer from plate with groove and protrusion using equivalent sand grain roughness and Stanton number [10]. The experimental work was done by J.Nikuradse who used a surface coated with closely packed sand grains of mean diameter $\boldsymbol{k}_{\boldsymbol{s}}$. As a consequence, other roughness patterns are often characterized in terms of equivalent sand grain roughness $\boldsymbol{k}_{\boldsymbol{s}}$ which gives the same friction factor in the fully rough regime. Whereas use of the equivalent sand grain roughness ensures that the correct friction factor is obtained in the fully rough regime [13].

\section{Assumptions}

This study is based on the following assumptions:

1. The fin material is homogeneous and isotropic.

2. The flow is steady.

3. The fluid is considered incompressible with constant properties.

4. The heat transfer coefficient is uniform over the fin surface.

5. There are no heat sources within the fin itself.

6. There is no free convection or radiation heat transfer.

\section{EXPERIMENTAL SET-UP}

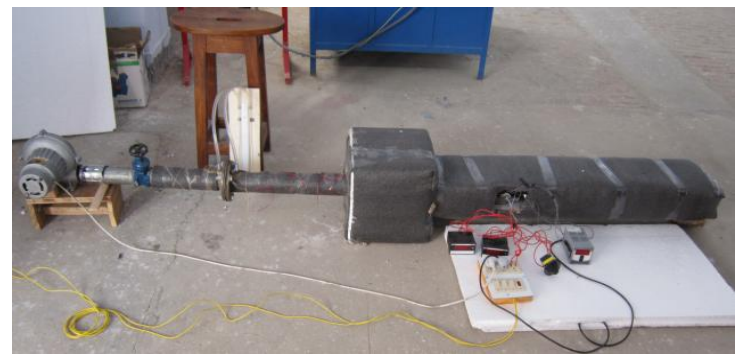

\section{Components of Experimental Set Up:-}

Rectangular Duct: The rectangular duct is made of ply wood. The duct is $110 \mathrm{~cm}$ long and have $18 \mathrm{~cm} \mathrm{X} 8 \mathrm{~cm}$ cross-section. One end is open to atmosphere and one end is connected to plenum. The duct is completely insulated by thermo-cole. The inlet section is given more length because we are required a fully developed boundary layer to remove the variations in mass flow rate of air because it alters the boundary layer at the entrance. Some length is provided to exit section because end effects will be minimized by doing so.

Test section: There is a test section in between the duct where the fins to be tested are placed. The test section cross section is $12 \mathrm{~cm} \mathrm{X} 8 \mathrm{~cm}$. Three fins will be kept inside one by one and they will have different geometries. Plenum: Plenum is a chamber in the middle of the duct and used to reduce shock waves. Plenum provides uniform flow in the duct and removes turbulence.

Heater: Electric heater is used to provide a constant heat flux to the aluminium plate. The heater is attached to the fin by fastener.

Thermocouples: Thermocouples are used at various position at inlet, outlet and on the surface to measure the temperature.

Temperature display device: This is a digital device used to display the temperature measured by thermocouple at various position. The temperature measured by instrument is in ${ }^{\circ} \mathrm{C}$.

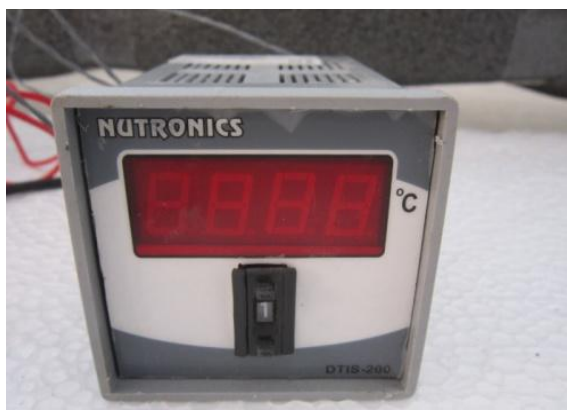


Voltmeter and ammeter: The digital voltmeter and ammeter is used to measure the value of voltage and current of input power to the heater. The voltmeter has a range of 440 volt and ammeter has a range of $1200^{\circ} \mathrm{C}$. The voltmeter and ammeter is used to measure the input power to the heater.

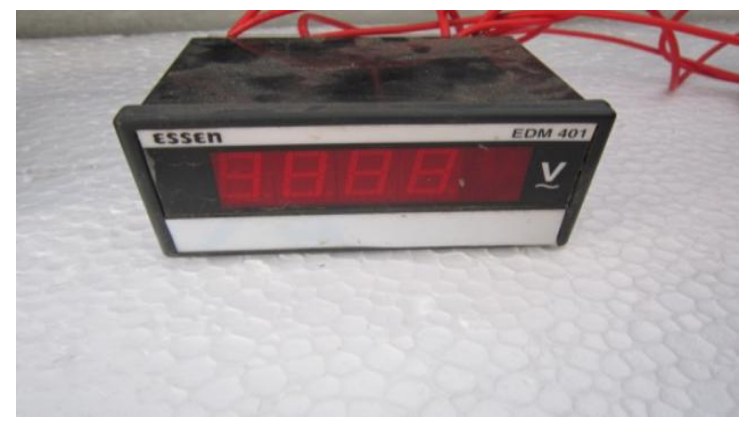

G.I. Pipe with flange: A flange is an external or internal rib, or rim (lip), for strength, as the flange of an iron beam or I-beam (or a T-beam); or for a flange of a train wheel; or for attachment to another object, as the flange on the end of a pipe, steam cylinder, etc., or on the lens mount of a camera. Thus a flanged rail is a rail with a flange on one side to keep wheels, etc., from running off. The term "flange" is also used for a kind of tool used to form flanges. Pipes with flanges can be assembled and disassembled easily.

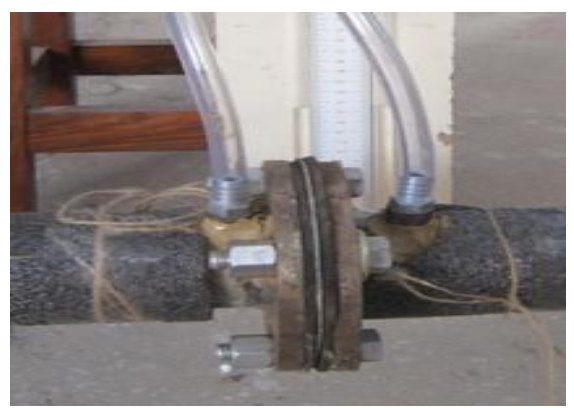

Orifice-meter and orifice plate: Orifice-meter is used to measure the mass flow rate and velocity of the fluid through duct. The most widely used flow metering principle involves placing a fixed area flow restriction of some type in the pipe or duct carrying the fluid. This flow restriction causes a pressure drop that varies with the flow rate. Thus, measurement of the pressure drop by means of a suitable differential-pressure pick up allows flow rate measurement.

$U$ - Tube manometer: Many techniques have been developed for the measurement of pressure and vacuum. Instruments used to measure pressure are called pressure gauges or vacuum gauges. A manometer could also be referring to a pressure measuring instrument, usually limited to measuring pressures near to atmospheric. The term manometer is often used to refer specifically to liquid column hydrostatic instruments.

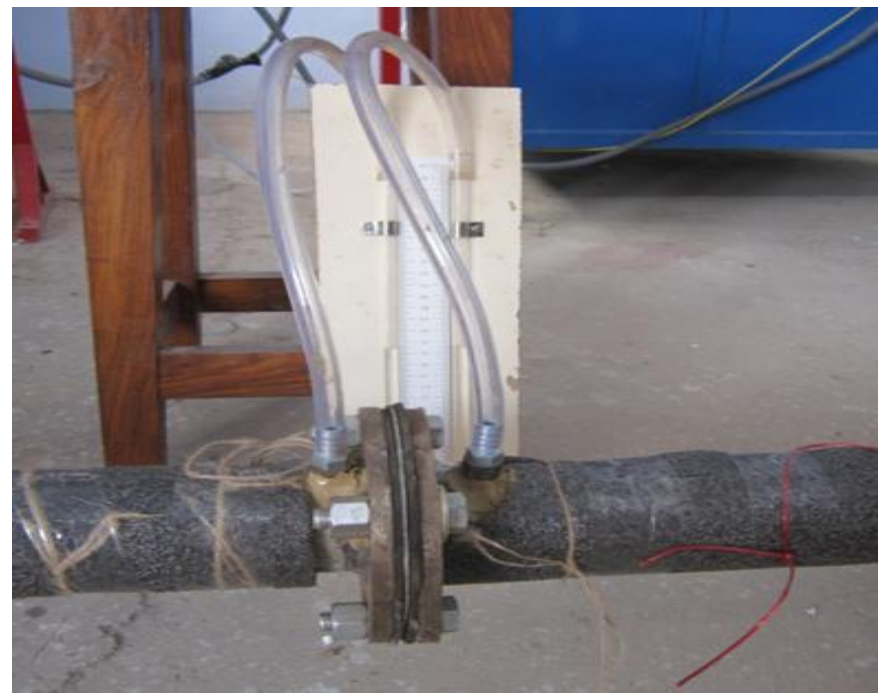


Centrifugal blower: A centrifugal blower is a mechanical device for moving air or other gases. It has a fan wheel composed of a number of fan blades, or ribs, mounted around a hub. As shown in Figure 1, the hub turns on a driveshaft that passes through the fan housing. The gas enters from the side of the fan wheel, turns 90 degrees and accelerates due to centrifugal force as it flows over the fan blades and exits the fan housing

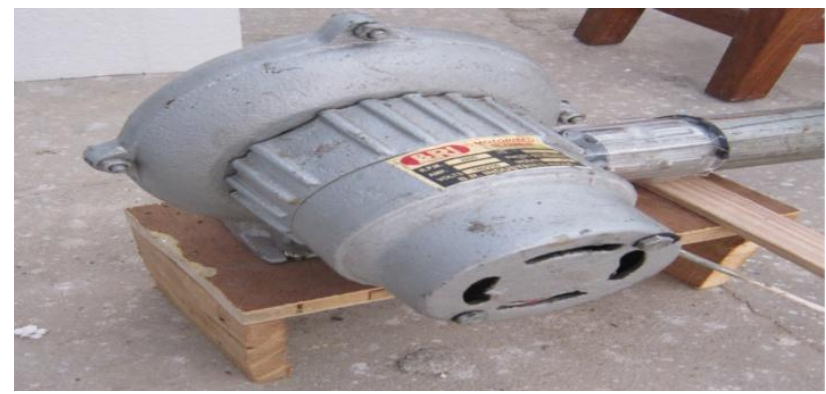

Aluminium fins: The three aluminium fins are used here to compare the rate of heat dissipation. The fins have the dimension of $12 \mathrm{~cm} \mathrm{X} 8 \mathrm{~cm} \mathrm{X} 0.5 \mathrm{~cm}$. The aluminium is used as a fin material because it is light in weight, have high conductivity, easily available and cheaper in comparison to copper.

Plane fin: This fin has a dimension of $12 \mathrm{~cm} \mathrm{X} 8 \mathrm{~cm} \mathrm{X} 0.5 \mathrm{~cm}$. There are three thermo couples on the surface of the fin to measure the surface temperature. The surface temperature is the average of three thermo couples.

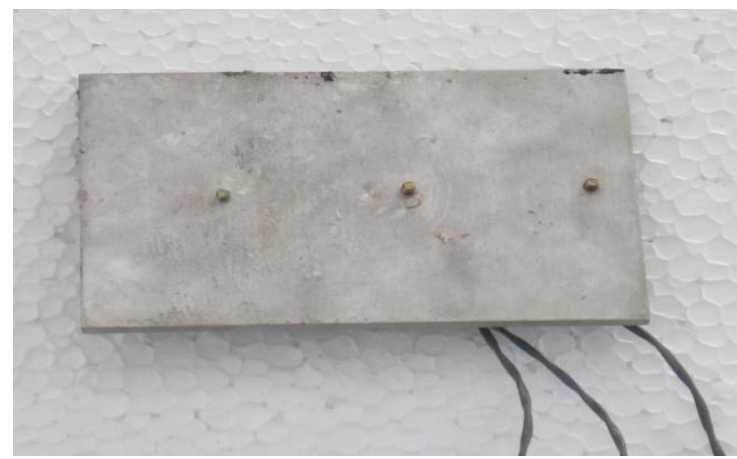

Protruded fin: This fin has the same dimension as plane fin but having a protrusion on the surface of the fin. Each protrusion has a diameter of $8 \mathrm{~mm}$ and height of $2.6 \mathrm{~mm}$. The pitch is $1.5 \mathrm{~cm}$ between two protrusions and the protrusions are in staggered way, so that maximum heat dissipation takes place.

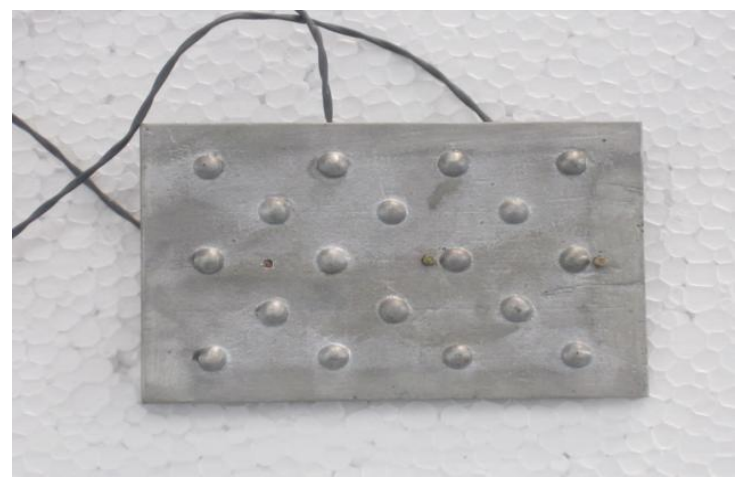

\section{EXPERIMENTAL PROCEDURE}

The aluminum plate is located in the middle of the test channel. Care is taken so that the aluminum plate can be located in the middle of the test section in order to ensure an equal channel height conditions and airflow rates for both sides of the aluminum plate. The blower is turned on and air is forced through the test setup. The flow rate through the test section is controlled with the help of a valve downstream of the orifice plate. The flow rate is set in such a way so that the pressure drop across the orifice corresponds to the required Reynolds number. After the flow is set across the test section, the heater is turned on and the voltage supplied to the heater is roughly set to $\mathbf{1 2 3 W}$. According to the temperature of the aluminum plate, voltage and current are changed. After the measured temperatures reached a specific value, the voltage is controlled to an input power 
123W. As time elapsed for roughly 45 minutes, the temperature of the aluminum plate reached steady state. The pressure difference across the orifice is checked frequently so that the flow rate would not change from the intended value of Reynolds number. At steady state, the temperatures of the aluminum plate are checked by the temperature indicator. The pressure difference across the orifice plate and the pressure across upstream of the orifice plate are measured. The voltage supplied to the heater and the corresponding current is taken to calculate the heat supplied to the aluminum plate. The temperatures of the inlet air into the test section and into the orifice plate are also checked. The above procedure is repeated for different mass flow rate and for different geometrical fins.

\section{DATA REDUCTION}

For plane rectangular fin

The Nusselt number for the flow over

flat plate can be calculated by the relation

$N u=0.664 \operatorname{Re}^{\frac{1}{2}} \operatorname{Pr}^{\frac{1}{3}}$

where $\operatorname{Re}=\frac{V l}{v}$

Convective heat transfer coefficient can be calculated by:

$$
N u=\frac{h l}{k}
$$

\section{For protruded fin}

Local skin friction coefficient is given as

$C f_{x}=\left(3.476+0.707 \ln \frac{x}{k_{s}}\right)^{-2.46}$

Average skin friction coefficient is given as

$C f=\left(2.635+0.618 \ln \frac{l}{k_{s}}\right)^{-2.57}$

Where

$k_{s}=2.48 \mathrm{~mm}$ for protrusion on plate

$$
(\dot{h}=2.6 \mathrm{~mm})
$$

The dimensionless characteristic

height is given by:

$\dot{h}^{+}=\left(\frac{k_{s}^{+}}{k_{s}}\right) \dot{h}$

The dimensionless sand grain size is given by:

$k_{s}^{+}=\frac{U_{e} k_{s}}{v_{e}} \sqrt{\frac{C f_{x}}{2}}$

The function $g\left(\dot{h}^{+}, P r\right)$ for protrusion is given by the relation:

$g\left(\dot{h}^{+}, \operatorname{Pr}\right)=0.55 \sqrt{h^{++}}\left(\operatorname{Pr}^{\frac{2}{3}}-1\right)+9.5$

Stanton number is given by the relation

$S t=\frac{C f / 2}{0.9+\sqrt{\frac{C f}{2}}\left[g\left(h^{+}, P r\right)-7.65\right]}$ 
We know

$S t=\frac{h}{\rho C p V}$

By this we find the value of $h$, convective heat transfer coefficient.

VII.

RESULT VALIDATION

Heat flux is given by the relation:

$\frac{Q}{A}=h \Delta T$

We know heat input is given by:

$Q=V I$

Therefore, $\quad \frac{V I}{A}=h\left(T_{s}-T_{\infty}\right)$

$\frac{V I}{t L}=h\left(T_{s}-T_{\infty}\right)$

As $t \ll L$

Therefore neglecting thickness

Then, $h=\frac{V I}{L\left(T_{s}-T_{\infty}\right)}$

VIII. EXPERIMENTAL RESULT:

\begin{tabular}{|c|c|c|c|c|}
\hline FIN TYPE & $\begin{array}{c}\text { Head, } \\
\mathbf{H}_{\mathbf{W}}\end{array}$ & $\mathbf{h}_{\text {experimental }}$ & $\mathbf{h}_{\text {actual }}$ & ERROR \\
\hline PLANE & 1.5 & 21.8 & 20.08 & $7.84 \%$ \\
\hline PLANE & 3 & 25.99 & 22.09 & $11.6 \%$ \\
\hline PROTRUDED & 1.5 & 23.24 & 25.35 & $8.35 \%$ \\
\hline PROTRUDED & 3 & 33.73 & 29.186 & $13.47 \%$ \\
\hline
\end{tabular}

\section{SUMMARY AND CONCLUSIONS}

\section{A. Summary}

Average convective heat transfer experiments were conducted using heater in experimental set up with rectangular duct. Total of eleven thermocouples are used to calculate the temperature more accurately. The three different fins are used to compare the heat transfer. These experiments were conducted to measure and calculate average heat transfer enhancement. The results of experiments are summarized as follows:

- The heat transfer through the plane fin is moderate and simple mathematical formulae of heat transfer through the surface can be used to calculate the heat transfer coefficient. But there is further scope of heat transfer enhancement from the surface.

- The blockages with circular protrusions enhance the heat transfer on the segment of rectangular channel wall downstream of the blockages, whereas they also cause pressure drops across two consecutive blockages. Therefore, heat transfer enhancement is beneficial but pressure drop is adverse compared to the heat transfer and heat transfer enhances if the mass flow rate is increased by regulating the valve.

\section{B. Conclusions}

For the experiments performed to study average heat transfer enhancement on different geometrical rectangular fins, the conclusions are as follows:

- The heat transfer through the plane fin is lesser than protruded fin. As flow just pass over the surface and extract the heat from the fin.

- The maximum heat transfer takes place from the protrusion fin due to the turbulence created by the geometrical blockages. In this case the mass flow rate corresponds to $3 \mathrm{~cm}$ head.

- The heat transfer here also depends on the pitch, and density of the protrusion. One thing more the flow pattern also depends on the protrusion position whether protrusion is inline or staggered. The heat transfer from the fin is maximum for staggered dense protrusion as the flow pattern is disturbed considerably in this case. 


\section{REFERENCES}

[1] Experimental investigation of heat transfer enhancement in radiating pin fin; Ganesh Murali j. Subrahmanya S. Katte

[2] Numerical study of heat transfer enhancement in a Plate fin heat exchanger using rectangular winglet type vortex generator; Munish Gupta

[3] Experimental investigation of heat transfer characteristics of cylindrical fin with different grooves; Mohammad Mashub, Zinat Rhman

[4] Recent Advances in heat transfer enhancements: AReview Report; M. Siddique, A.Y.Boukhary

[5] Experimental investigation of high performance thermal module with dimple vortex generators; Kai-Shing Yang, Chi-Chuan Wang

[6] Experimental investigation of heat transfer enhancement from dimpled surface in a channel; Sandeep s. Kore, Narayan K. Sane

[7] Shape Optimization of a plate heat exchanger with a dimpled surface; Jonghyeok Lee, Se-Jin Yook

[8] An experimental study of endwall heat transfer enhancement for flow past staggered non-conducting pin fin array; Vamsee Satish Achanta

[9] Enhancement of natural convection heat transfer from a fin by triangular perforation of base parallel and toward its base; Abdullah H. AlEssa and Mohammed Q AL Odat

[10] Heat Transfer; Pearson Education; A.F. Mills and V.Ganesan

[11] On the skin friction coefficient for a fully rough flat plate; Mills,A.F, Xu Hang

[12] Heat and Mass transfer between a rough wall and turbulent fluid flow at high Reynolds and Peclet numbers; Yaglom, A.M. and Kader,B.A.

[13] Law of flow in rough pipes; Nikuradse,J..

[14] Heat Transfer Enhancement Techniques; Prof Alina Adriana Minea Technical University Gh. Asachi Iasi 